\title{
A theory for non-Newtonian viscoelastic polymeric liquids
}

\author{
Lallit Anand* \\ Department of Mechanical Engineering \\ Massachusetts Institute of Technology \\ Cambridge, MA 02139, USA
}

May 3, 2016

\begin{abstract}
In many existing theories for incompressible polymeric liquids the Cauchy stress is decomposed as $\mathbf{T}=$ $-p \mathbf{1}+\mathbf{S}^{v}+\mathbf{S}^{e}$, where $p$ is an arbitrary pressure, $\mathbf{S}^{v}=2 \mu_{s} \mathbf{D}$ a deviatoric viscous stress with $\mu_{s}$ a viscosity and $\mathbf{D}$ the deviatoric stretching tensor, and $\mathbf{S}^{e}$ is a deviatoric elastic stress which is introduced to account for stiffening arising from the alignment of long-chain polymer molecules during flow. A constitutive equation for $\mathbf{S}^{e}$ needs to be prescribed and there are a large number of different proposals in the literature, with most proposals involving a hypoelastic rate constitutive equation for $\mathbf{S}^{e}$ given in terms of a suitable frame-indifferent rate, which is usually taken as the Oldroyd or upper-convected rate. As is well-known, a hypoelastic equation for the stress is not thermodynamically consistent, in the sense that the constitutive equation for $\mathbf{S}^{e}$ is not derived form a free energy function.

The purpose of this paper is to present an alternative - thermodynamically-consistent and frameindifferent - continuum theory for incompressible viscoelastic liquids. The theory is based on a Kröner-type multiplicative decomposition of the deformation gradient $\mathbf{F}$ of the form $\mathbf{F}=\mathbf{F}^{e} \mathbf{F}^{p}$. In this theory the elastic stress $\mathbf{S}^{e}$ is derived from a free-energy function which is prescribed in terms of a suitable measure based on the unimodular elastic distortion tensor $\mathbf{F}^{e}$. This relation is supplemented by an evolution equation for the unimodular plastic distortion tensor $\mathbf{F}^{p}$ - the plastic flow rule.

We study the response of the constitutive theory in steady simple shearing and steady extensional flows. We show: (i) that the theory qualitativley reproduces the experimentally-observed transient shear-thinning and normal stress effects during shearing flows of a polymer melt; and (ii) that it also reproduces the transient extensional response of a polymer melt.
\end{abstract}

Keywords: B. polymeric material; viscoelastic material; viscoplastic material.

\section{Introduction}

An accurate mathematical description of the large deformations of polymer melts and concentrated polymer solutions, both of which are viscoelastic liquids, is important for the development of a numerical simulation capability for polymer processing operations, and also for simulating the behavior of various bodily fluids in human physiology. Viscoelastic liquids are capable of accumulating large recoverable strains during flow — which puts such materials in an intermediate position between liquids and solids - and this makes their rheological behavior quite complex.

In the past four decades there has been substantial progress in formulating theories for such materials, and reviews of the literature may be found in textbooks and several review articles (cf., e.g., Giesekus, 1982; Bird et al., 1987a,b; Bird and Wiest, 1995; Leonov, 1999; Tanner, 2000; McKinley and Sridhar, 2002; Larson and Desai, 2015). For isothermal and incompressible fluid flows, most current theories are described by:

*Tel.: +1-617-253-1635; E-mail address: anand@mit.edu 
- Incompressibility constraint,

$$
\operatorname{div} \mathbf{v}=0
$$

with $\mathbf{v}$ the velocity.

- Balance of linear and angular momentum,

$$
\operatorname{div} \mathbf{T}+\mathbf{b}_{0}=\rho \dot{\mathbf{v}}, \quad \mathbf{T}=\mathbf{T}^{\top},
$$

where $\mathbf{T}$ is the Cauchy stress, $\mathbf{b}_{0}$ the non-inertial body force per unit volume, and $\rho$ the constant mass density.

- Constitutive equation for $\mathbf{T}$,

$$
\mathbf{T}=-p \mathbf{1}+\mathbf{S}, \quad \text { with } \quad \operatorname{tr} \mathbf{S}=0
$$

where $p$ is an arbitrary hydrostatic pressure, and $\mathbf{S}$ the deviatoric extra stress, which is given by

$$
\left.\begin{array}{rl}
\mathbf{S} & =\mathbf{S}^{v}+\mathbf{S}^{e}, \\
\mathbf{S}^{v} & =2 \mu_{s} \mathbf{D} \quad \text { - viscous extra stress, } \\
\mathbf{S}^{e} & \text { - elastic extra stress, }
\end{array}\right\}
$$

where, $\mathbf{D}$ is the stretching tensor, $\mu_{s}$ is viscosity of the purely viscous contribution. The elastic contribution is denoted by $\mathbf{S}^{e}$.

A constitutive equation or $\mathbf{S}^{e}$ needs to be prescribed, and there are a bewilderingly large number of different proposals in the literature. The classical proposal is due to Oldroyd (1950), in which $\mathbf{S}^{e}$ is taken to evolve according to the differential equation

$$
\stackrel{\diamond}{\mathbf{S}^{e}}=2 G \mathbf{D}-\frac{1}{\lambda_{r}} \mathbf{S}^{e}, \quad \text { with initial condition }\left.\quad \mathbf{S}^{e}\right|_{t=0}=\mathbf{0},
$$

in which

$$
\stackrel{\diamond}{\mathbf{S}}^{e}=\dot{\mathbf{S}}^{e}-\mathbf{L S}^{e}-\mathbf{S}^{e} \mathbf{L}^{\top}
$$

is the frame-indifferent upper-convected rate, also known as Oldroyd rate of $\mathbf{S}^{e}, G$ is a constant elastic shear modulus, and $\lambda_{r}$ is constant relaxation time. ${ }^{1}$

- The set of constitutive equations (1.3) through (1.6) is known as the Oldroyd-B model for nonNewtonian fluids.

A simple derivation based on hypoelastic theories of viscoplasticity of solids, ${ }^{2}$ which leads to the classical Oldroyd-B model is as follows. The stretching tensor $\mathbf{D}$ is additively decomposed into an elastic part $\mathbf{D}^{e}$ and a viscoplastic part $\mathbf{D}^{p}$ as

$$
\mathbf{D}=\mathbf{D}^{e}+\mathbf{D}^{p}
$$

and $\mathbf{S}^{e}$ is given as a rate evolution equation of the form

$$
\grave{\mathbf{S}}^{e}=2 G \mathbf{D}^{e}=2 G\left(\mathbf{D}-\mathbf{D}^{p}\right)
$$

with $G$ an elastic shear modulus. The plastic stretching $\mathbf{D}^{p}$ is given by a flow rule, which is formulated as follows. Let

$$
\mathbf{N}^{p}=\frac{\mathbf{D}^{p}}{\left|\mathbf{D}^{p}\right|}, \quad \text { whenever } \quad \mathbf{D}^{p} \neq \mathbf{0},
$$

denote the plastic flow direction. This is taken to be codirectional with the stress $\mathbf{S}^{e}$, in the sense that

$$
\mathbf{N}^{p}=\frac{\mathbf{S}^{e}}{\left|\mathbf{S}^{e}\right|}
$$

\footnotetext{
${ }^{1}$ In the non-Newtonian fluids literature one also finds use of many other frame-indifferent rates of $\mathbf{S}^{e}$; the choice of a frame-indifferent rate is not unique.

${ }^{2}$ Such theories are well-known to Plasticians, especially readers of the International Journal of Plasticity.
} 
so that

$$
\mathbf{D}^{p}=\left|\mathbf{D}^{p}\right| \frac{\mathbf{S}^{e}}{\left|\mathbf{S}^{e}\right|} \quad \text { whenever } \mathbf{S}^{e} \neq \mathbf{0} .
$$

Next, defining an equivalent plastic shear strain rate by ${ }^{3}$

$$
\dot{\bar{\gamma}}^{p} \stackrel{\text { def }}{=} \sqrt{2}\left|\mathbf{D}^{p}\right|
$$

and an equivalent shear stress for plastic flow (based on $\mathbf{S}^{e}$ ) by

$$
\bar{\tau} \stackrel{\text { def }}{=} \frac{1}{\sqrt{2}}\left|\mathbf{S}^{e}\right|
$$

eq. (1.10) may be written as Mises-type flow rule

$$
\mathbf{D}^{p}=\dot{\bar{\gamma}}^{p}\left(\frac{\mathbf{S}^{e}}{2 \bar{\tau}}\right) \quad \text { for } \bar{\tau} \neq 0 .
$$

As a final ingredient, the elastic equivalent shear stress $\bar{\tau}$ is taken to be balanced by a shear stress $g\left(\dot{\bar{\gamma}}^{p}\right)$ which is a function of plastic equivalent shear strain rate,

$$
\bar{\tau}=g\left(\dot{\bar{\gamma}}^{p}\right)
$$

We call (1.14), which represents a microforce balance, the strength relation. The function $g(\cdot)$ is a positive-valued function of $\dot{\bar{\gamma}}^{p}$ which satisfies a dissipation inequality

$$
g\left(\dot{\bar{\gamma}}^{p}\right) \dot{\bar{\gamma}}^{p}>0 \quad \text { whenever } \quad \dot{\bar{\gamma}}^{p}>0 .
$$

Thus, using (1.14) in (1.13) and introducing a shear-rate dependent plastic viscosity

$$
\eta^{p}=\hat{\eta}^{p}\left(\dot{\bar{\gamma}}^{p}\right) \stackrel{\text { def }}{=} \frac{g\left(\dot{\bar{\gamma}}^{p}\right)}{\dot{\bar{\gamma}}^{p}},
$$

we may write the plastic stretching $\mathbf{D}^{p}$ as

$$
\mathbf{D}^{p}=\frac{\mathbf{S}^{e}}{2 \eta^{p}},
$$

substitution of which in (1.8) gives

$$
\stackrel{\diamond}{\mathbf{S}}^{e}+\frac{1}{\lambda_{r}} \mathbf{S}^{e}=2 G \mathbf{D}
$$

where

$$
\lambda_{r}=\hat{\lambda}_{r}\left(\dot{\bar{\gamma}}^{p}\right) \stackrel{\text { def }}{=} \frac{\hat{\eta}^{p}\left(\dot{\bar{\gamma}}^{p}\right)}{G}
$$

is a shear-rate dependent relaxation time. Thus unlike the classical Oldroyd-B model given in (1.5), in the simple derivation outlined above the relaxation time $\lambda_{r}$ is not a constant, but a function of $\dot{\bar{\gamma}}^{p}$.

There are many well-known models in the complex fluids literature — such as the the Cross model (Cross, 1965) and the Carreau-Yasuda model (Yasuda et al., 1981) — which may be used for the shear-ratedependent strength function $g\left(\dot{\bar{\gamma}}^{p}\right)$ in the generalized Oldroyd-B model described above. ${ }^{4}$ This generalized Oldroyd-B model can represent:

(i) shear-thinning (or shear-thickening) behavior by a suitable choice of the function $g\left(\dot{\bar{\gamma}}^{p}\right)$;

(ii) an incrementally linear response through the elastic constant $G$;

(iii) a strain-rate-dependent relaxation time; and

\footnotetext{
${ }^{3}$ Use of the notation $\dot{\bar{\gamma}}^{p}$ for the equivalent plastic shear strain rate is traditional. Here, a superposed dot does not represent a material time derivative.

${ }^{4}$ We use the adjective "generalized" because in the classical Oldroyd-B model the relation $g\left(\dot{\bar{\gamma}}^{p}\right)$ is linear in $\dot{\bar{\gamma}}^{p}$, which gives a constant relaxation time.
} 
(iv) it is properly frame-indifferent since it uses the Oldroyd-rate (1.8).

However, since it uses a hypoelastic relation (1.8),

- it is not thermodynamically consistent, in the sense that this constitutive equation for $\mathbf{S}^{e}$ is not derived form a free energy function; also

- it is unclear how to generalize it in a consistent fashion to account for large elastic stretches.

Accordingly, the purpose of this paper is to present an alternative, thermodynamically-consistent and frame-indifferent, continuum theory for incompressible viscoelastic liquids. In our theory the elastic stress $\mathbf{S}^{e}$ arises due to a change in the entropy of the material, as dominantly determined by the statistical effects of changes in the internal molecular conformations, much as in classical continuum theories of non-linear elasticity of elastomeric materials. However, unlike elastomeric materials in which the polymeric chains are chemically cross-linked, in polymeric liquids it is the mechanical entanglements that serve as temporary cross-links which get destroyed (and reformed) during deformation, as the entangled polymer molecules slide relative to each other. The viewpoint that polymeric liquids have much in common with elastomeric materials is due to Lodge (1964), who argued that both types of materials consist of densely packed long chain molecules, and both exist only at temperatures well above their glass transition temperatures. The major difference between the structures of polymeric liquids and elastomers is that the cross-links are chemical and (almost) permanent in elastomers but temporary in polymeric liquids — with the temporary cross-links being caused by the entanglement of the long chain polymer molecules.

In contrast to the addiditive decomposition $\mathbf{D}=\mathbf{D}^{e}+\mathbf{D}^{p}$, used above in the derivation of the generalized Oldroyd-B model, our theory is based on a multiplicative decomposition of the deformation gradient $\mathbf{F}$ of the form (Kröner, 1960; Lee, 1969),

$$
\mathbf{F}=\mathbf{F}^{e} \mathbf{F}^{p}
$$

The elastic stress $\mathbf{S}^{e}$ is derived from a free-energy function which is prescribed in terms of a suitable measure based on the elastic distortion $\mathbf{F}^{e}$. This relation is supplemented by an evolution equation for the plastic distortion $\mathbf{F}^{p}$ - the plastic flow rule.

The decomposition (1.20) is widely used in theories of elasto-viscoplasticity of metals, as well as that of amorphous polymers below their glass transition temperatures (cf., e.g., Gurtin et al., 2010; Boyce et al., 1988; Arruda and Boyce, 1993a; Anand and Gurtin, 2003). A multi-mechanism version of (1.20), F = $\mathbf{F}^{e(\alpha)} \mathbf{F}^{p(\alpha)}(\alpha=1, \ldots, M)$, has also been successfully used for modeling the behavior of glassy polymers, both below and above their glass transition temperatures (cf., e.g., Boyce et al., 2000; Dooling et al., 2002). In a set of papers we have used a similar framework to model the response of solid polymeric materials (Anand et al., 2009; Ames et al., 2009; Srivastava et al., 2010a,b). In this paper we extend our recent work on solid polymers to model the non-Newtonian response of viscoelastic polymeric liquids.

The theory is mathematically involved and so as not to burden readers who are not interested in details, we relegate its formal development to the Supplementary Material to this paper. The theory is summarized in Section 2. In Section 3 we study the response of the theory in steady simple shearing and extensional flows. We close in Section 4 with some final remarks.

Before closing this section we note that Rajagopal and Srinivasa (2000) have previously used an elasticplastic multiplicative decomposition of the deformation gradient to formulate a rate-type theory for viscoelastic liquids. There are many shared features between their theory and the one formulated in this paper. However we emphasize that the procedures and details of development of their theory by Rajagopal and Srinivasa (2000), are different in many details from the detailed theory presented as Supplementary Material to our paper. Further, Rajagopal and Srinivasa (2000) do not show how their theory would respond in simple shearing and extensional flows, as we do here in Section 3. 


\section{Constitutive theory}

The basic fields which lead to constitutive equations for the viscous stress $\mathbf{S}^{v}$ and the elastic stress $\mathbf{S}^{e}$ are: ${ }^{5}$

$$
\begin{array}{ll}
\mathbf{F}=\nabla \boldsymbol{\chi}, \quad \operatorname{det} \mathbf{F}=1, & \text { deformation gradient; } \\
\mathbf{L}=\operatorname{grad} \mathbf{v}=\mathbf{\mathbf { F }} \mathbf{F}^{-1}, \quad \operatorname{tr} \mathbf{L}=0, & \text { velocity gradient; } \\
\mathbf{D}=\operatorname{sym} \mathbf{L}, \quad \operatorname{tr} \mathbf{D}=0, & \text { stretching; } \\
\mathbf{F}=\mathbf{F}^{e} \mathbf{F}^{p} & \text { decomposition of } \mathbf{F} ; \\
\mathbf{F}^{e}, \quad \operatorname{det} \mathbf{F}^{e}=1, & \text { elastic distortion; } \\
\mathbf{F}^{p}, \quad \operatorname{det} \mathbf{F}^{p}=1, & \text { plastic distortion; } \\
\mathbf{B}^{e}=\mathbf{F}^{e} \mathbf{F}^{e}, & \text { elastic left Cauchy-Green tensor; } \\
I_{1}\left(\mathbf{B}^{e}\right)=\operatorname{tr} \mathbf{B}^{e}, & \text { first invariant of } \mathbf{B}^{e} ; \\
I_{2}\left(\mathbf{B}^{e}\right)=\frac{1}{2}\left[\left(\operatorname{tr} \mathbf{B}^{e}\right)^{2}-\operatorname{tr}\left(\mathbf{B}^{e 2}\right)\right], & \text { second invariant of } \mathbf{B}^{e} ; \\
\psi_{\mathrm{I}}=\bar{\psi}_{\mathrm{I}}\left(I_{1}\left(\mathbf{B}^{e}\right), I_{2}\left(\mathbf{B}^{e}\right)\right), & \text { free-energy density per unit volume } \\
& \text { of the intermediate space. }
\end{array}
$$

The special set of constitutive equations considered here consist of:

\section{Viscous stress:}

With

$$
\dot{\bar{\gamma}} \stackrel{\text { def }}{=} \sqrt{2}|\mathbf{D}|
$$

defining a scalar equivalent shearing rate, the viscous stress $\mathbf{S}^{v}$ is taken to be given by

$$
\mathbf{S}^{v}=2 \mu_{s} \mathbf{D}
$$

with $\mu_{s}$ a solvent viscosity.

\section{The free-energy:}

where with

$$
\psi_{\mathrm{I}}=\tilde{\psi}_{\mathrm{I}}\left(\bar{\lambda}^{e}\right)+\frac{1}{2} C \ln \left(\frac{I_{2}}{3}\right)
$$

$$
\bar{\lambda}^{e} \stackrel{\text { def }}{=} \frac{1}{\sqrt{3}} \sqrt{\operatorname{tr} \mathbf{B}^{e}}
$$

denoting an effective elastic stretch, the first term in (2.3) accounts for the dependence of $\psi_{\mathrm{I}}$ on the first invariant of $\mathbf{B}^{e}$, while the second term with $C>0$ a constant, accounts for the dependence of the free-energy on the second invariant of $\mathbf{B}^{e}$.

In polymeric materials the major part of the free-energy $\tilde{\psi}_{\mathrm{I}}\left(\bar{\lambda}^{e}\right)$ arises from an "entropic" contribution. Based on statistical mechanics models of rubber elasticity (cf., e.g. Treloar, 1975; Arruda and Boyce, 1993b) we consider the Langevin-inverse form

$$
\begin{aligned}
\tilde{\psi}_{\mathrm{I}}\left(\bar{\lambda}^{e}\right) & =G_{R} \lambda_{L}^{2}\left(\left(\frac{\bar{\lambda}^{e}}{\lambda_{L}}\right) x+\ln \left(\frac{x}{\sinh x}\right)-\left(\frac{1}{\lambda_{L}}\right) y-\ln \left(\frac{y}{\sinh y}\right)\right), \\
x & =\mathcal{L}^{-1}\left(\frac{\bar{\lambda}^{e}}{\lambda_{L}}\right), \quad y=\mathcal{L}^{-1}\left(\frac{1}{\lambda_{L}}\right),
\end{aligned}
$$

\footnotetext{
${ }^{5}$ We use standard notation of modern continuum mechanics (Gurtin et al., 2010). Specifically: $\nabla$ and Div denote the gradient and divergence with respect to the material point $\mathbf{X}$ in the reference configuration, and $\Delta=\operatorname{Div} \nabla$ denotes the referential Laplace operator; grad div, and div grad denote these operators with respect to the point $\mathbf{x}=\boldsymbol{\chi}(\mathbf{X}, t)$ in the deformed body. As is usual, we use a superposed dot for the material time-derivative, e.g. for $\phi(\mathbf{x}, t)$ a scalar field,

$$
\dot{\phi}(\mathbf{x}, t)=\frac{\partial}{\partial t} \phi(\mathbf{x}, t)+\mathbf{v}(\mathbf{x}, t) \cdot \operatorname{grad} \phi(\mathbf{x}, t),
$$

where $\mathbf{v}$ is the velocity. Throughout, we write $\mathbf{F}^{e-1}=\left(\mathbf{F}^{e}\right)^{-1}, \mathbf{F}^{e-\top}=\left(\mathbf{F}^{e}\right)^{-\top}$, etc. We write tr $\mathbf{A}$, sym $\mathbf{A}$, skw $\mathbf{A}, \mathbf{A} 0$, and $\operatorname{sym}_{0} \mathbf{A}$ respectively, for the trace, symmetric, skew, deviatoric, and symmetric-deviatoric parts of a tensor $\mathbf{A}$. Occasionaly we will also write $\operatorname{dev} \mathbf{A}$ to denote the deviatoric part of $\mathbf{A}$. Also, the inner product of tensors $\mathbf{A}$ and $\mathbf{B}$ is denoted by $\mathbf{A}$ : $\mathbf{B}$, and the magnitude of $\mathbf{A}$ by $|\mathbf{A}|=\sqrt{\mathbf{A}: \mathbf{A}}$.
} 
where $\mathcal{L}^{-1}$ is the inverse of the Langevin function

$$
\mathcal{L}(\cdots)=\operatorname{coth}(\cdots)-(\cdots)^{-1} .
$$

This free-energy involves two material parameters:

$$
\begin{aligned}
& G_{R}=N k_{B} \vartheta, \text { called the rubbery modulus, and } \\
& \lambda_{L}=\sqrt{n} \text { called the network locking stretch. }
\end{aligned}
$$

Here, $N$ is he number of chains per unit volume of the intermediate space, and $n$ is the number of links in each chain. Also, $k_{B}$ is Boltzmann's constant, and $\vartheta$ is the constant temperature for the isothermal theory under consideration.

\section{Elastic stress:}

The elastic stress $\mathbf{S}^{e}$ is given by

$$
\mathbf{S}^{e}=G \operatorname{dev}\left(\mathbf{B}^{e}\right)+\frac{C}{I_{2}} \operatorname{dev}\left(I_{1} \mathbf{B}^{e}-\left(\mathbf{B}^{e}\right)^{2}\right)
$$

where $G$ is a stretch-dependent shear modulus given by

$$
G=G_{R}\left(\frac{\lambda_{L}}{3 \bar{\lambda}^{e}}\right) \mathcal{L}^{-1}\left(\frac{\bar{\lambda}^{e}}{\lambda_{L}}\right) .
$$

Since $\mathcal{L}^{-1}(x) \rightarrow \infty$ as $x \rightarrow 1$, the modulus $G \rightarrow \infty$ as $\bar{\lambda}^{e} \rightarrow \lambda_{L}$.

\section{Mandel stress:}

The symmetric Mandel stress $\mathbf{M}^{e}$ which governs plastic flow is given by

$$
\mathbf{M}^{e}=\mathbf{F}^{e \top} \mathbf{S}^{e} \mathbf{F}^{e-\top}
$$

in terms of which the equivalent shear stress for plastic flow is defined by

$$
\bar{\tau} \stackrel{\text { def }}{=} \frac{1}{\sqrt{2}}\left|\mathbf{M}_{0}^{e}\right|
$$

\section{Evolution equations for $\mathbf{F}^{p}$ :}

The evolution equation for each $\mathbf{F}^{p}$, the plastic flow rule, is ${ }^{6}$

$$
\begin{aligned}
\dot{\mathbf{F}}^{p} & =\mathbf{D}^{p} \mathbf{F}^{p},\left.\quad \mathbf{F}^{p}\right|_{t=0}=\mathbf{1}, \\
\mathbf{D}^{p} & =\dot{\bar{\gamma}}^{p}\left(\frac{\mathbf{M}_{0}^{e}}{2 \bar{\tau}}\right)
\end{aligned}
$$

${ }^{6}$ The plastic stretching in (2.12) is based on the $\mathbf{F}=\mathbf{F}^{e} \mathbf{F}^{p}$ decomposition, and it is not the same as that in (1.7) which is directly based on an additive decomposition of $\mathbf{D}$. 
with $\dot{\bar{\gamma}}^{p}$ determined by solving the strength equation

$$
\bar{\tau}=g\left(\dot{\bar{\gamma}}^{p}\right)
$$

where $g\left(\dot{\bar{\gamma}}^{p}\right)$ is a positive-valued function which satisfies the dissipation inequality

$$
g\left(\dot{\bar{\gamma}}^{p}\right) \dot{\bar{\gamma}}^{p}>0 \quad \text { whenever } \quad \dot{\bar{\gamma}}^{p}>0 .
$$

\section{Response of the theory in steady simple shearing and exten- sional flows}

\subsection{Steady simple shearing flow}

Consider a steady simple shearing flow defined by

$$
\mathbf{v}(\mathbf{x})=\mathbf{L}(\mathbf{x}-\mathbf{o}),
$$

with respect to an origin $\mathbf{o}$, where

$$
\mathbf{L}=\dot{\gamma} \mathbf{e}_{1} \otimes \mathbf{e}_{2},
$$

is a spatially constant velocity gradient with respect to a Cartesian basis $\left(\mathbf{e}_{1}, \mathbf{e}_{2}, \mathbf{e}_{3}\right)$, with

$$
\dot{\gamma} \text { a constant shear strain rate. }
$$

In steady simple shearing flow experiments on viscoelastic liquids it has been found that a shear stress alone does not suffice to determine simple shearing; additional normal stresses arise. In experiments, one can measure the shear stress component $T_{12}$ of the Cauchy stress $\mathbf{T}$, and two normal-stress component differences $\left(T_{11}-T_{22}\right)$ and $\left(T_{22}-T_{33}\right)$, and these may be expressed as

$$
\begin{aligned}
& T_{12}=\eta \dot{\gamma}, \\
& N_{1} \stackrel{\text { def }}{=} T_{11}-T_{22}=\Psi_{1} \dot{\gamma}^{2}, \\
& N_{2} \stackrel{\text { def }}{=} T_{22}-T_{33}=\Psi_{2} \dot{\gamma}^{2},
\end{aligned}
$$

so that $T_{12}$ is an odd function of $\dot{\gamma}$, while the normal-stress differences $N_{1}$ and $N_{2}$ are even functions of $\dot{\gamma}$; the normal-stress differences are unchanged when the sign of shear strain rate is reversed. Experiments show that (cf., e.g., Bird and Wiest, 1995):

- The non-Newtonian viscosity $\eta$ is positive for all liquids, and it is usually found to be a monotonically decreasing function of the shear rate $\dot{\gamma}$.

- The first normal stress coefficient $\Psi_{1}$ has been found to be positive for nearly all polymeric liquids, and it decreases very rapidly with increasing shear rate.

- The second normal-stress coefficient $\Psi_{2}$, has been found to be negative. Measurements of $\Psi_{2}$ are quite sparse, because of experimental difficulties.

Fig. 1, extracted from the PhD thesis by Kraft (1996), shows experimentally-measured data for the transient viscosity $\eta$, as well as the first normal stress coefficient $\Psi_{1}$, at various shearing rates $\dot{\gamma}$ in the range $10^{-3}$ through $10 \mathrm{~s}^{-1}$, for a low density polyethylene melt (LDPE, Lupolen $1810 \mathrm{H}$ ) at $150^{\circ} \mathrm{C}$. 


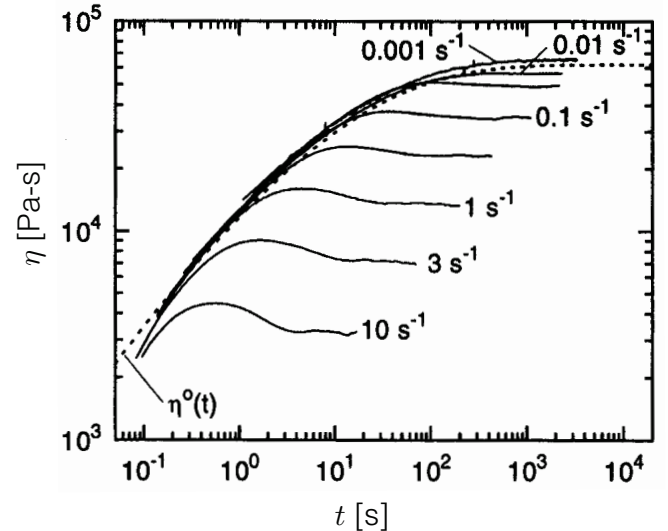

(a)

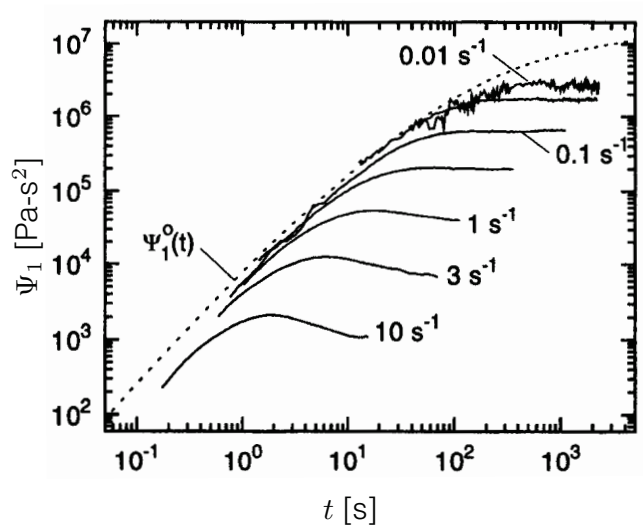

(b)

Figure 1: Experimentally-measured transient (a) viscosity $\eta$, and (b) first normal stress function $\Psi_{1}$, for a LDPE (Lupolen $1810 \mathrm{H})$ melt at $150^{\circ} \mathrm{C}$, at various shearing rates $\dot{\gamma}$ in the range $10^{-3}$ through $10 \mathrm{~s}^{-1}$. From Kraft (1996).

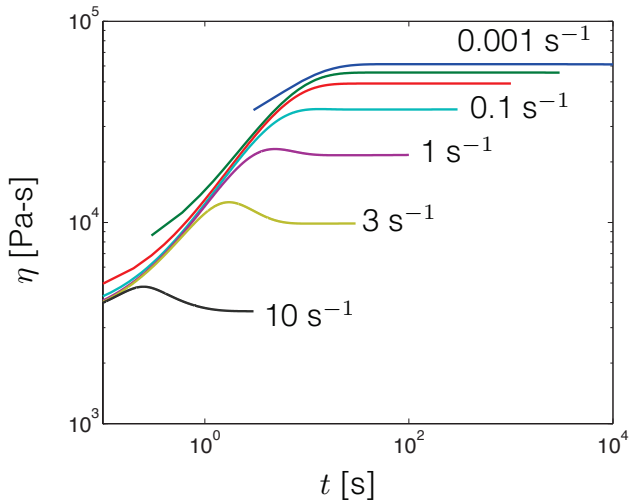

(a)

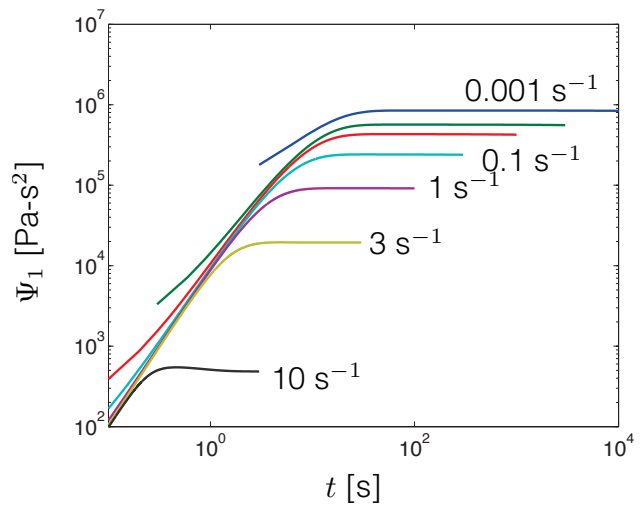

(b)

Figure 2: Theoretically-predicted transient (a) viscosity $\eta$, and (b) first normal stress function $\Psi_{1}$, for $\mathrm{LDPE}$ (Lupolen $1810 \mathrm{H}$ ) at $150^{\circ} \mathrm{C}$ at various shearing rates $\dot{\gamma}$ in the range $10^{-3}$ through $10 \mathrm{~s}^{-1}$.

Fig. 2 shows the corresponding transient viscosity $\eta$ and the first normal stress coefficient $\Psi_{1}$ predicted by the theory formulated in this paper. The results shown in this figure were obtained by using the following material parameters/functions: ${ }^{7}$

- The viscosity $\mu_{s}$ in $(2.2)$ is taken as $\mu_{s}=3 \mathrm{kPa}-\mathrm{s}$.

- The elastic constants $G_{R}$ and $\lambda_{L}$ in (2.7) are taken as $G_{R}=10 \mathrm{kPa}$ and $\lambda_{L}=10$, while the constant $C$ in (2.3) was set to zero so that effects of the second invariant of $\mathbf{B}^{e}$ are neglected. $^{8}$

- Finally, the strength relation (2.13) was taken in the form

$$
\bar{\tau}=\eta^{p} \dot{\bar{\gamma}}^{p} \quad \text { with } \quad \eta^{p}=\frac{\eta_{0}}{1+\left(\zeta \dot{\bar{\gamma}}^{p}\right)^{m}}
$$

${ }^{7}$ All calculations reported here were carried out using a simple, semi-implicit time-integration procedure programmed in Matlab. The time-integration procedure is a variant of the well-known elastic-viscoplastic time-integration procedure presented in Weber and Anand (1990). Since the procedure used here is semi-implicit and not fully-implicit, one has to be careful about the size of the time-step used in the numerical calculations.

${ }^{8}$ In the absence of viscous effects, the special case $C=0$ corresponds to the widely-used Arruda-Boyce model of finite elasticity for elastomeric materials (Arruda and Boyce, 1993b). 
where the plastic viscosity $\eta^{p}$ is taken in the classical Cross (1965) form, with material a

$$
\eta_{0}=60 \mathrm{kPa}-\mathrm{s}, \quad \zeta=4.7 \mathrm{~s}, \quad m=0.65 .
$$

Remark. Since the generalized shear modulus G, cf. eq. (2.9), goes to infinity as the effective elastic stretch $\bar{\lambda}^{e}$ approaches the locking stretch $\lambda_{L}$, in our numerical calculations we have limited the value of $\mathrm{G}$ to its value when $\bar{\lambda}^{e}=0.95 \lambda_{L}$.

A plot of the plastic viscosity $\eta^{p}$ as a function of the equivalent plastic shear strain rate $\dot{\bar{\gamma}}^{p}$ according to (3.5) showing shear-thinning behavior is shown in Fig. 3.

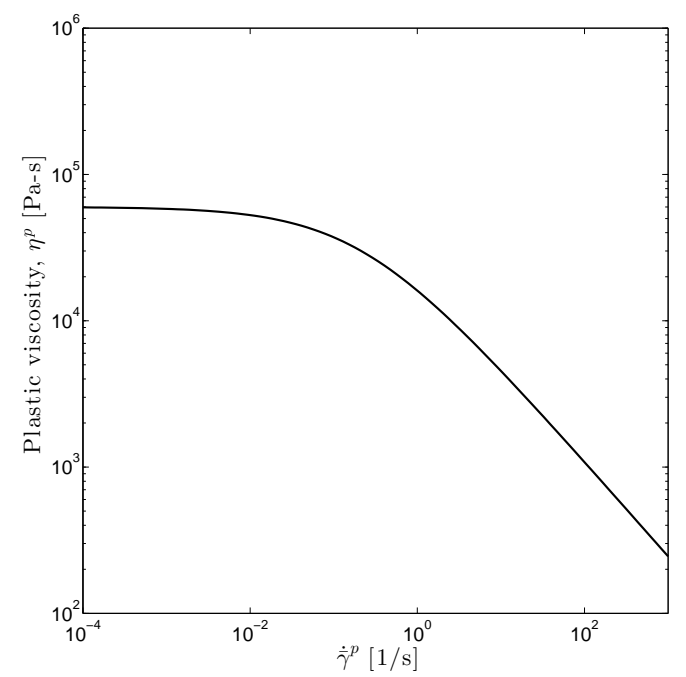

Figure 3: A plot of the plastic viscosity $\eta^{p}$ as a function of the equivalent plastic shear strain rate $\dot{\bar{\gamma}}^{p}$ showing shear-thinning behavior according to (3.5).

The plots for the transient viscosity and the first normal stress coefficient shown in Fig. 2, are qualitatively similar to the experimental curves shown in Fig. 1. The response shown in Fig. 2, which was obtained using only six material parameters, is very encouraging.

\subsection{Steady extensional flow}

Next, consider a steady incompressible extensional flow defined by a spatially constant velocity gradient

$$
\mathbf{L}=\dot{\epsilon}\left(\mathbf{e}_{1} \otimes \mathbf{e}_{1}-0.5 \mathbf{e}_{2} \otimes \mathbf{e}_{2}-0.5 \mathbf{e}_{3} \otimes \mathbf{e}_{3}\right),
$$

with $\dot{\epsilon}$ a constant extensional strain rate. ${ }^{9}$ The extensional viscosity in such a flow is defined by

$$
\eta_{E}^{+} \stackrel{\text { def }}{=} \frac{T_{11}}{\dot{\epsilon}}
$$

Fig. 4 shows the transient extensional viscosity predicted by the theory for the LDPE (Lupolen $1810 \mathrm{H}$ ) melt at $150^{\circ} \mathrm{C}$. Kraft (1996) does not report on extensional viscosities in his thesis, but Sentmanat et al. (2005) show results for another LDPE (Lupolen $1840 \mathrm{H}$ ) melt at $150^{\circ} \mathrm{C}$. The transient extensional response shown in Fig. 4 is qualitatively similar to that reported by Sentmanat et al. (2005) in their Fig. 7.

\footnotetext{
${ }^{9}$ Use of the notation $\dot{\epsilon}$ for the extensional strain rate is traditional. Here, a superposed dot does not represent a material time derivative.
} 


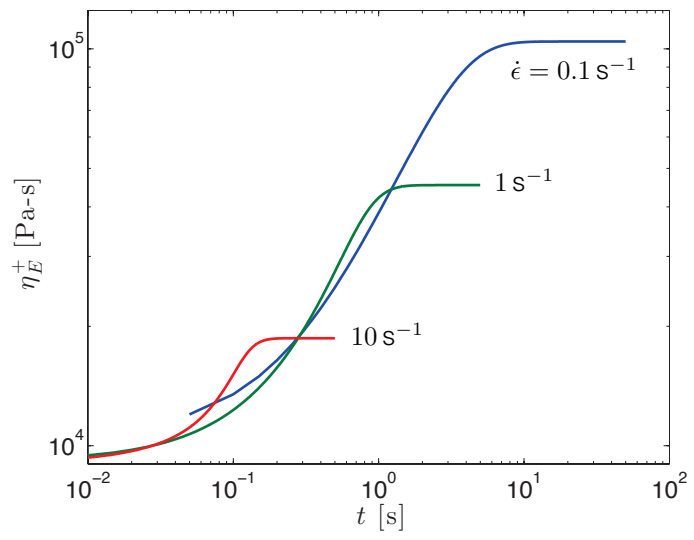

Figure 4: Theoretically-predicted transient extensional viscosity $\eta_{E}^{+}$for LDPE (Lupolen $1810 \mathrm{H}$ ) at $150^{\circ} \mathrm{C}$ at extensional rates $\dot{\epsilon}$ of $0.1,1$ and $10 \mathrm{~s}^{-1}$.

We emphasize that it is not the purpose of this paper to present results of elaborate curve-fitting procedures to match experimental data, but instead to present a general framework of the theory, and show that this theory - when suitably specialized - reproduces the major experimentally-observed trends for viscoelastic liquids.

Indeed, most polymeric liquids are composed of polymer molecules of different lengths. This "polydispersity" strongly affects the rheological properties of these materials (cf., e.g., Bird and Wiest, 1995). Accordingly, a "multi-mode" approach is often found to be necessary to reproduce the experimentallymeasured stress-strain response over a broad range of strain rates (cf., e.g., Verbeeten et al., 2002). In an Appendix, Section 5, we summarize a multi-mode version of the single-mode theory described above. This generalized theory, with many more material parameters, might give predictions which are in better accord with experimental data.

\section{Concluding remarks}

- Constitutive equations for fluids are typically phrased such that they are independent of the choice of reference configuration. The theory for viscoelastic liquids formulated here does not have this characteristic. The Cauchy stress is given by $\mathbf{T}(\mathbf{x}, t)=-p(\mathbf{x}, t) \mathbf{1}+\mathbf{S}^{v}(\mathbf{x}, t)+\mathbf{S}^{e}(\mathbf{x}, t)$. While the constitutive equation for the viscous stress $\mathbf{S}^{v}(\mathbf{x}, t)$ in our theory depends only on the deviatoric stretching $\mathbf{D}(\mathbf{x}, t)$ which is defined in the deformed body, the constitutive equation for the elastic stress $\mathbf{S}^{e}(\mathbf{x}, t)$ depends on the distortion $\mathbf{F}^{e}(\mathbf{X}, t)$ which is given by $\mathbf{F}^{e}(\mathbf{X}, t)=\mathbf{F}(\mathbf{X}, t)\left(\mathbf{F}^{p}(\mathbf{X}, t)\right)^{-1}$.

- We refrain from taking a rate of the elastic stress $\mathbf{S}^{e}$ and trying to ensure that the rate is properly frameindifferent. There is no need to do so, our theory satisfies the principle of material frame-indifference.

- In our theory all normal stress differences in simple shearing flows arise because of our nonlinear elastic relation for $\mathbf{S}^{e}$, which is derived from a free-energy based on a statistical mechanics model of entropic elasticity. ${ }^{10}$

- Particular forms for the strength relation (2.13) in the visocoplastic flow rule will benefit from additional detailed molecular-level cosiderations of thermal activation (Eyring, 1936), as well as molecular-level considerations of slip dynamics of entangled polymer chains (Doi and Edwards, 1986). Here, in lieu of such detailed molecular-level considerations, we have used a simple phenomenological form (3.5) due to Cross (1965), which is widely-known to have practical utility.

\footnotetext{
${ }^{10}$ We have neglected a $\mathbf{D}^{2}$ dependence of the viscous stress $\mathbf{S}^{v}$, and this assumption precludes any normal stress differences in simple shearing flows from arising due to the constitutive equation for $\mathbf{S}^{v}$.
} 
- Much work needs to be done to account for the molecular architecture of the constituent molecules (molecular weight, chain stiffness, chain branching) and how these govern the "viscoplastic mechanisms" such as the cumulative effects of destruction of temporary mechanical cross-links and changes in resistance to relative chain slippage of the long-chain polymer molecules under imposed deformation.

As in many existing viscoplasticity theories for metals, as well as solid polymers (cf., e.g., Gurtin et al., 2010; Anand et al., 2009; Ames et al., 2009; Srivastava et al., 2010a,b), the framework described above may be augmented by introducing additional internal variables, accompanied by suitable evolution equations, to represent the hardening/softening response due to chain-slippage viscoplastic mechanisms.

- Flows of interest in polymer processing operations are markedly nonisothermal; the theory needs to be extended to account for heat conduction and for thermomechanical coupling effects.

\section{Acknowledgements}

Support from NSF (CMMI Award No. 019646-001) is gratefully acknowledged.

\section{Appendix. Multimode version of the theory}

In order to obtain a realistic description of the stress-strain response of viscoelastic liquids over a broad range of strain rates, a "multi-mode" approach has often been found to be necessary (Verbeeten et al., 2002). In this Appendix we summarize a multi-mode version of the single-mode theory proposed in the main body of the paper. The multi-mode theory is based on a generalized Kröner-type decomposition of the deformation gradient $\mathbf{F}$ of the form,

$$
\mathbf{F}=\mathbf{F}^{e(\alpha)} \mathbf{F}^{p(\alpha)}, \quad \alpha=1, \ldots, M .
$$

In what follows, when convenient, we use the shorthand notation

$$
\sum_{\alpha}=\sum_{\alpha=1}^{M} .
$$

In the multimode theory, the basic fields which lead to constitutive equations for the viscous stress $\mathbf{S}^{v}$ and the elastic stress $\mathbf{S}^{e}$ are:

$$
\begin{array}{ll}
\mathbf{F}=\nabla \boldsymbol{\chi}, \quad \operatorname{det} \mathbf{F}=1, \quad & \text { deformation gradient; } \\
\mathbf{L}=\operatorname{grad} \mathbf{v}=\dot{\mathbf{F}} \mathbf{F}^{-1}, \quad \operatorname{tr} \mathbf{L}=0, & \text { velocity gradient; } \\
\mathbf{D}=\operatorname{sym} \mathbf{L}, \quad \operatorname{tr} \mathbf{D}=0, & \text { stretching; } \\
\mathbf{F}=\mathbf{F}^{e(\alpha)} \mathbf{F}^{p(\alpha)}, \quad \alpha=1, \ldots, M & \text { multimechanism decomposition of } \mathbf{F} ; \\
\mathbf{F}^{e(\alpha)}, \quad \operatorname{det} \mathbf{F}^{e(\alpha)}=1, & \text { elastic distortions; } \\
\mathbf{F}^{p(\alpha)}, \quad \operatorname{det} \mathbf{F}^{p(\alpha)}=1, & \text { plastic distortions; } \\
\mathbf{B}^{e(\alpha)}=\mathbf{F}^{e(\alpha)} \mathbf{F}^{e(\alpha) \top}, & \text { elastic left Cauchy-Green tensors; } \\
I_{1}\left(\mathbf{B}^{e(\alpha)}\right)=\operatorname{tr} \mathbf{B}^{e(\alpha)}, & \text { first invariant of } \mathbf{B}^{e(\alpha)} ; \\
I_{2}\left(\mathbf{B}^{e(\alpha)}\right)=\frac{1}{2}\left[\left(\operatorname{tr} \mathbf{B}^{e(\alpha)}\right)^{2}-\operatorname{tr}\left(\mathbf{B}^{e(\alpha) 2}\right)\right], & \text { second invariant of } \mathbf{B}^{e(\alpha)} ; \\
\psi_{\mathrm{I}}=\sum_{\alpha} \bar{\psi}_{\mathrm{I}}^{(\alpha)}\left(I_{1}\left(\mathbf{B}^{e(\alpha)}\right), I_{2}\left(\mathbf{B}^{e(\alpha)}\right)\right), & \text { free-energy density. }
\end{array}
$$

The special set of constitutive equations consist of:

\section{Viscous stress:}

With

$$
\dot{\bar{\gamma}} \stackrel{\text { def }}{=} \sqrt{2}|\mathbf{D}|
$$

defining a scalar equivalent shearing rate, we take $\mathbf{S}^{v}$ to be given by

$$
\mathbf{S}^{v}=2 \mu_{s} \mathbf{D}
$$

with $\mu_{s}$ a solvent viscosity. 


\section{The elastic free-energy:}

We assume a non-interacting separable free energy of the form

$$
\psi_{\mathrm{I}}=\sum_{\alpha} \tilde{\psi}_{\mathrm{I}}^{(\alpha)}\left(\bar{\lambda}^{e(\alpha)}\right)+\sum_{\alpha} \frac{1}{2} C^{(\alpha)} \ln \left(\frac{I_{2}\left(\mathbf{B}^{e(\alpha)}\right)}{3}\right),
$$

where with

$$
\bar{\lambda}^{e(\alpha)} \stackrel{\text { def }}{=} \frac{1}{\sqrt{3}} \sqrt{\operatorname{tr} \mathbf{B}^{e(\alpha)}}
$$

denoting an effective elastic stretch for the $\alpha$ th micromechanism, the first term in (5.4) accounts for the dependence of $\psi_{\mathrm{I}}$ on the first invariant of $\mathbf{B}^{e(\alpha)}$, while the second term with $C^{(\alpha)}>0$ a constant, accounts for the dependence of the free-energy on the second invariant of $\mathbf{B}^{e(\alpha)}$.

The contribution to the free-energy from the first invariant of $\mathbf{B}^{e(\alpha)}$ for each micromechanism is taken to be given by

$$
\begin{aligned}
\tilde{\psi}_{\mathrm{I}}^{(\alpha)}\left(\bar{\lambda}^{e(\alpha)}\right) & =G_{R}^{(\alpha)} \lambda_{L}^{(\alpha) 2}\left(\left(\frac{\bar{\lambda}^{e(\alpha)}}{\lambda_{L}^{(\alpha)}}\right) x+\ln \left(\frac{x}{\sinh x}\right)-\left(\frac{1}{\lambda_{L}^{(\alpha)}}\right) y-\ln \left(\frac{y}{\sinh y}\right)\right), \\
x & =\mathcal{L}^{-1}\left(\frac{\bar{\lambda}^{e(\alpha)}}{\lambda_{L}^{(\alpha)}}\right), \quad y=\mathcal{L}^{-1}\left(\frac{1}{\lambda_{L}^{(\alpha)}}\right),
\end{aligned}
$$

where $\mathcal{L}^{-1}$ is the inverse of the Langevin function. This free-energy function involves two material parameters for each $\alpha$ :

- $G_{R}^{(\alpha)}=N^{\alpha} k_{B} \vartheta$, called the rubbery modulus, and

- $\lambda_{L}^{(\alpha)}=\sqrt{n^{\alpha}}$ called the network locking stretch.

Here, $N^{\alpha}$ are the number of chains per unit volume of the intermediate space for the $\alpha$ th mechanism, and $n^{\alpha}$ are the number of links in each chain.

3. Elastic stress: The elastic stress $\mathbf{S}^{e}$ is given by

$$
\mathbf{S}^{e}=\sum_{\alpha} \mathbf{S}^{e(\alpha)}
$$

with

$$
\mathbf{S}^{e(\alpha)}=G^{(\alpha)} \operatorname{dev}\left(\mathbf{B}^{e(\alpha)}\right)+\frac{C^{(\alpha)}}{I_{2}\left(\mathbf{B}^{e(\alpha)}\right)} \operatorname{dev}\left(I_{1}\left(\mathbf{B}^{e(\alpha)}\right) \mathbf{B}^{e(\alpha)}-\left(\mathbf{B}^{e(\alpha)}\right)^{2}\right),
$$

and with the moduli $G^{(\alpha)}$ given by

$$
G^{(\alpha)}=G_{R}^{\alpha}\left(\frac{\lambda_{L}^{(\alpha)}}{3 \bar{\lambda}^{e(\alpha)}}\right) \mathcal{L}^{-1}\left(\frac{\bar{\lambda}^{e(\alpha)}}{\lambda_{L}^{(\alpha)}}\right) .
$$

Remark. It is possible to derive the additivity of the elastic stresses from the various micromechanisms, eq. (5.8), by using the principle of virtual power, in a manner similar to that which has been followed in the formulation of the theory in the Supplmentary Material to this paper.

\section{Mandel stresses:}

The Mandel stress $\mathbf{M}^{(\alpha)}$ which govern the plastic flow of each mechanism is given by

$$
\mathbf{M}^{(\alpha)}=\mathbf{F}^{e(\alpha) \top} \mathbf{S}^{e(\alpha)} \mathbf{F}^{e(\alpha)-\top} .
$$

Also

$$
\bar{\tau}^{(\alpha)} \stackrel{\text { def }}{=} \frac{1}{\sqrt{2}}\left|\mathbf{M}_{0}^{(\alpha)}\right|
$$

defines an equivalent plastic shear stress for the $\alpha$ th micromechanism. 


\section{Evolution equation for $\mathbf{F}^{p(\alpha)}$ :}

The evolution equation for each $\mathbf{F}^{p(\alpha)}$, the flow rule for the $\alpha$ th micromechanism, is

$$
\dot{\mathbf{F}}^{p(\alpha)}=\mathbf{D}^{p(\alpha)} \mathbf{F}^{p(\alpha)},\left.\quad \mathbf{F}^{p(\alpha)}\right|_{t=0}=\mathbf{1}
$$

with

$$
\mathbf{D}^{p(\alpha)}=\dot{\bar{\gamma}}^{p(\alpha)}\left(\frac{\mathbf{M}_{0}^{(\alpha)}}{2 \bar{\tau}^{(\alpha)}}\right),
$$

and with $\dot{\bar{\gamma}}^{p(\alpha)}$ determined by solving the strength relations

$$
\bar{\tau}^{(\alpha)}=g^{(\alpha)}\left(\dot{\bar{\gamma}}^{p(\alpha)}\right)
$$

\section{References}

Anand, L., Gurtin, M.E., 2003. A theory of amorphous solids undergoing large deformations, with applications to polymeric glasses. International Journal of Solids and Structures 40, 1465-1487.

Anand, L., Ames, N.M., Srivastava, V., Chester, S.A., 2009. A thermo-mechanically coupled theory for large deformations of amorphous polymers. Part I: Formulation. International Journal of Plasticity 25, 1474-1494.

Ames, N.M., Srivastava, V., Chester, S.A., Anand, L., 2009. A thermo-mechanically coupled theory for large deformations of amorphous polymers. Part II: Applications. International Journal of Plasticity 25, 1495-1539.

Arruda, E. M., Boyce, M.C., 1993a. Evolution of plastic anisotropy in amorphous polymers during finite straining, International Journal of Plasticity 9, 697-720.

Arruda, E. M., Boyce, M.C., 1993b. A three-dimensional constitutive model for the large stretch behavior of rubber elastic materials. Journal of the Mechanics and Physics of Solids 41, 389-412.

Bird, R.A., Armstrong, R. C., Hassager, O., Dynamics of Polymeric Liquids. Volume 1: Fluid Mechanics Wiley Interscience, 1987.

Bird, R.A., Armstrong, R. C., Hassager, O., Dynamics of Polymeric Liquids. Volume 2: Kinetic Theory Wiley Interscience, 1987.

Bird, R.A., Weist, J.M., 1995. Constitutive equations for polymeric liquids. Annual Reviews of Fluid Mechanics 27, 169-193.

Boyce, M.C. and Parks, D.M., Argon, A. S., 1988. Large inelastic deformation of glassy polymers. Part 1: rate dependent constitutive model. Mechanics of Materials 7, 15-33.

Boyce, M. C., Socrate, S., Llana, P. G., 2000. Constitutive model for the finite deformation stress-strain behavior of poly(ethylene terephthalate) above the glass transition. Polymer, 41, 2183-2201.

Cross, M.M., 1965. Rheology of non-Newtonian fluids: a new flow equation for psuedoplastic fluids. Journal of Colloid Science 20, 417-437.

Doi, M., Edwards, S. F., 1986. The Theory of Polymer Dynamics. Oxford University Press.

Dooling, P. J., Buckley, C. P., Rostami, S., Zahalan, N., 2002. Hot-drawing of poly(methyl metahcrylate) and simulation using a glass-rubber consitutive model. Polymer 43, 2451-2465.

Eyring, H., 1936. Viscosity, Plasticity, and Diffusion as Examples of Absolute Reaction Rates. Journal of Chemical Physics 4, 283-291. 
Giesekus, H., 1982. A simple constitutive equation for polymer fluids based on the concept of deformationdependent tensorial mobility. Journal of Non-Newtonian Fluid Mechanics 11, 69-109.

Gurtin, M.E., Fried, E., Anand, L., 2010. The Mechanics and Thermodynamics of Continua. Cambridge University Press, Cambridge.

M. Kraft, 1996. Untersuchungen zur scherinduzierten rheologischen Anisotropievon verschiedenen Polyethylen-Schmelzen. PhD Dissertation No. 11417, ETH Zurich.

Kröner, E., 1959. Allgemeine kontinuumstheorie der versetzungen und eigenspannungen. Archive for Rational Mechanics and Analysis 4, 273-334.

Lee, E. H., Elastic plastic deformation at finite strain, ASME Journal of Applied Mechanics 36 (1969) 1-6.

Larson, R.G., Desai, P.S., 2015. Modeling the rheology of polymer melts and solutions. Annual Reviews of Fluid Mechanics 47, 47-65.

Leonov, A. I., 1999. Constitutive equations for viscoelastic liquids: formulation, analysis and comparison with data. Advances in the Flow and Rheology of Non-Newtonian Fluids, Part A, edited by Siginer, D. A., Kee, D. De, Chhabra, R. P., Elsevier, 1999, 519-575. 85-98.

Lodge, A.S., 1964. Elastic liquids. Academic Press, London-N.Y.

McKinley, G.H., Sridhar, T., 2002. Filament-stretching rheometry of complex fluids. Annual Reviews of Fluid Mechanics 34, 375-415.

Oldroyd, J.G., 1950. On the formulation of rheological equations of state. Proceedings of the Royal Society London A200, 523-541.

Sentmanat, M., Wang, B.N., McKinley, G.H., 2005. Measuring the transient extensional rheology of polyethylene melts using the SER universal testing platform. Journal of Rheology 49, 585-606.

Srivastava, V., Chester, S.A., Ames, N.M., Anand, L., 2010. A thermo-mechanically-coupled largedeformation theory for amorphous polymers in a temperature range which spans their glass transition. International Journal of Plasticity 26, 1138-1182.

Srivastava, V., Chester, S.A., Anand, L., 2010. Thermally actuated shape-memory polymers: Experiments, theory, and numerical simulations. Journal of the Mechanics and Physics of Solids 58, 1100-1124.

Tanner, R.I., 2000. Engineering Rheology, Oxford.

Rajagopal, K.R., and Srinivasa, A. R., 2000. A thermodynamic frame-work for rate type fluid models, Journal of Non-Newtonian Fluid Mechanics 88, 207-227.

Treloar, L.R.G., 1975. The Physics of Rubber Elasticity, Oxford.

Verbeeten, W.M.H., Peters, G.W.M., Baaijens, F.P.T., 2002. Viscoelastic analysis of complex polymer melt flows using the eXtended Pom-Pom model. Journal of Non-Newtonian Fluid Mechanics 108, 310-236.

Weber, G. and Anand, L., 1990. Finite deformation constitutive equations and a time integration procedure for isotropic, hyperelastic-viscoplastic solids. Computer Methods In Applied Mechanics and Engineering 79, 173-202.

Yasuda, K., Armstrong, R.C., Cohen, R.E., 1981. Shear flow properties of concentrated solutions of linear and star branched polystyrenes. Rheologica Acta 20, 163-178. 